\title{
Supporting Information: High-Throughput Detection of Thiamine using Periplasmic Binding Protein-based Biorecognition
}

Katie A. Edwards ${ }^{1,2}$, Woo Jin Seog ${ }^{2}$, Lu Han ${ }^{3}$, Seth Feder ${ }^{4}$, Clifford E. Kraft ${ }^{1}$, Antje J. Baeumner ${ }^{2}$

${ }^{1}$ Department of Natural Resources, Cornell University, Ithaca, NY

${ }^{2}$ Department of Biological and Environmental Engineering, Cornell University, Ithaca, NY

${ }^{3}$ Department of Food Science, Cornell University, Ithaca, NY

${ }^{4}$ Department of Chemical Engineering, Cornell University, Ithaca, NY

\section{Table of contents}

Liposome preparation and phospholipid concentration analysis: ...................................................

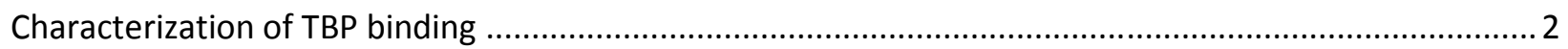

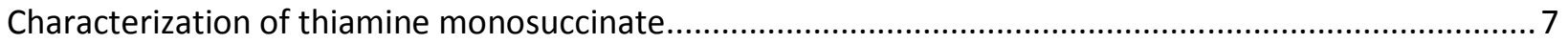

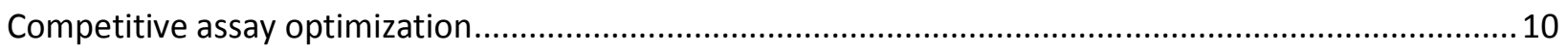

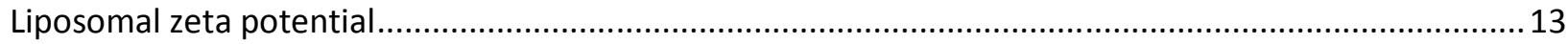

Comparison to commercially available thiamine ELISA ................................................................... 14

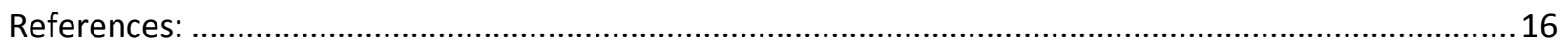




\section{Liposome preparation and phospholipid concentration analysis:}

Sulforhodamine B (SRB)-encapsulating liposomes were prepared using the reverse phase evaporation method $^{1,2}$ as described previously using 36.2 mol\% DPPC, 17.8 mol\% DPPG, 45.9 mol\% cholesterol, and 6 mol\% N-glutaryl DPPE. ${ }^{3} 150$ mM SRB was prepared in $20 \mathrm{mM}$ 4-Morpholineethane-sulfonic acid sodium salt (MES), pH 6.5 and 20 mM MES, pH 6.5 containing $0.2 \mathrm{M}$ sodium chloride and $0.01 \%(\mathrm{w} / \mathrm{v}$ ) sodium azide (MESS) for size exclusion chromatography and dialysis. 1-ethyl-3-(3-dimethylaminopropyl) carbodiimide (EDC)-mediated conjugation (5-15 molar equivalents to the total carboxylic acid functionalized lipid input) to TBP at 0.05 mol\% was carried out as described previously. ${ }^{3}$ The phospholipid content for each liposome batch was determined using the Bartlett assay ${ }^{4,5}$, with the procedure carried out as previously described. ${ }^{3}$

\section{Characterization of TBP binding}

TBP binding was characterized using equilibrium filtration assays where TBP in the presence or absence of thiamine was incubated in the presence of various solution conditions, then the mixture was centrifuged through a defined MWCO membrane chosen to retain TBP and any bound thiamine while allowing free thiamine to filter through. Subsequently, the amount of thiamine remaining unbound in the filtrate was assessed.

No thiamine binding was observed at $\mathrm{pH} 3.0$, and a slight decrease was observed at 4.0 and $\mathrm{pH}$ 8.0, though intermediate $\mathrm{pH}$ values yielded consistent binding (Fig. S1). The addition of casein and low concentrations of Tween-20 seemed to promote binding, whereas there were no significant trends with sodium chloride concentrations (Fig. S2). 


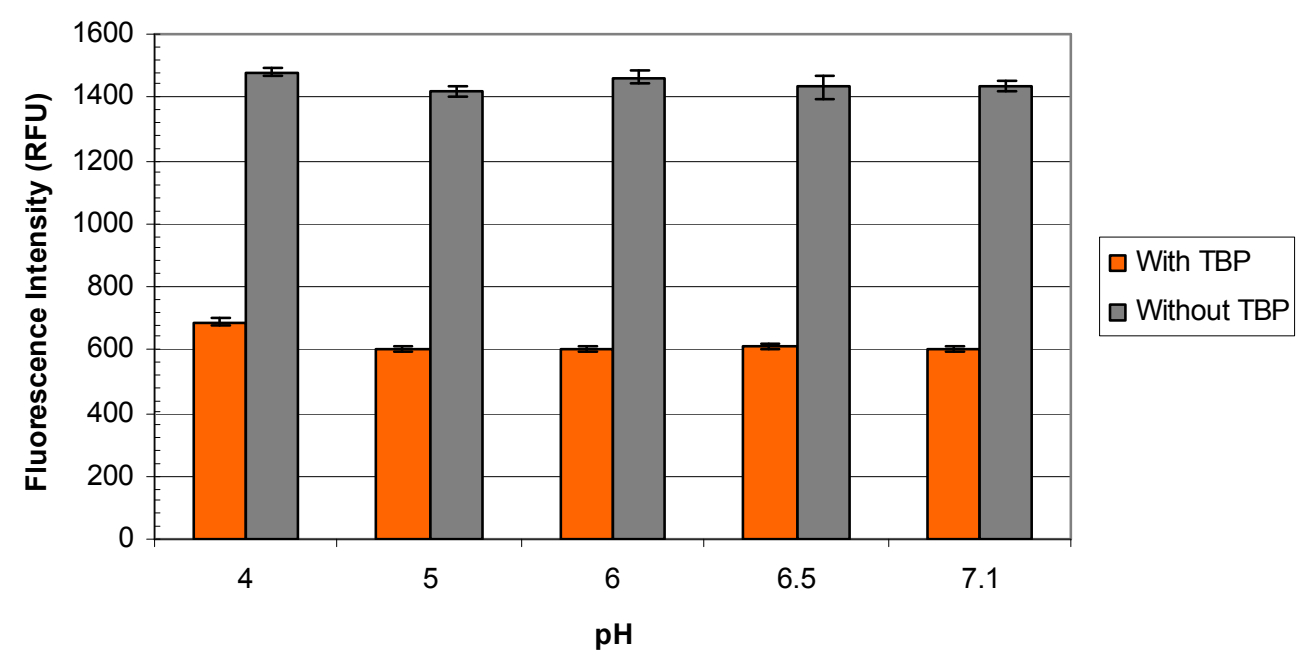

Figure S1. Effect of $\mathrm{pH}$ on TBP binding to thiamine using equilibrium filtration assays. Each bar represents the average of triplicate determinations with error bars representing the standard deviation of these measurements.

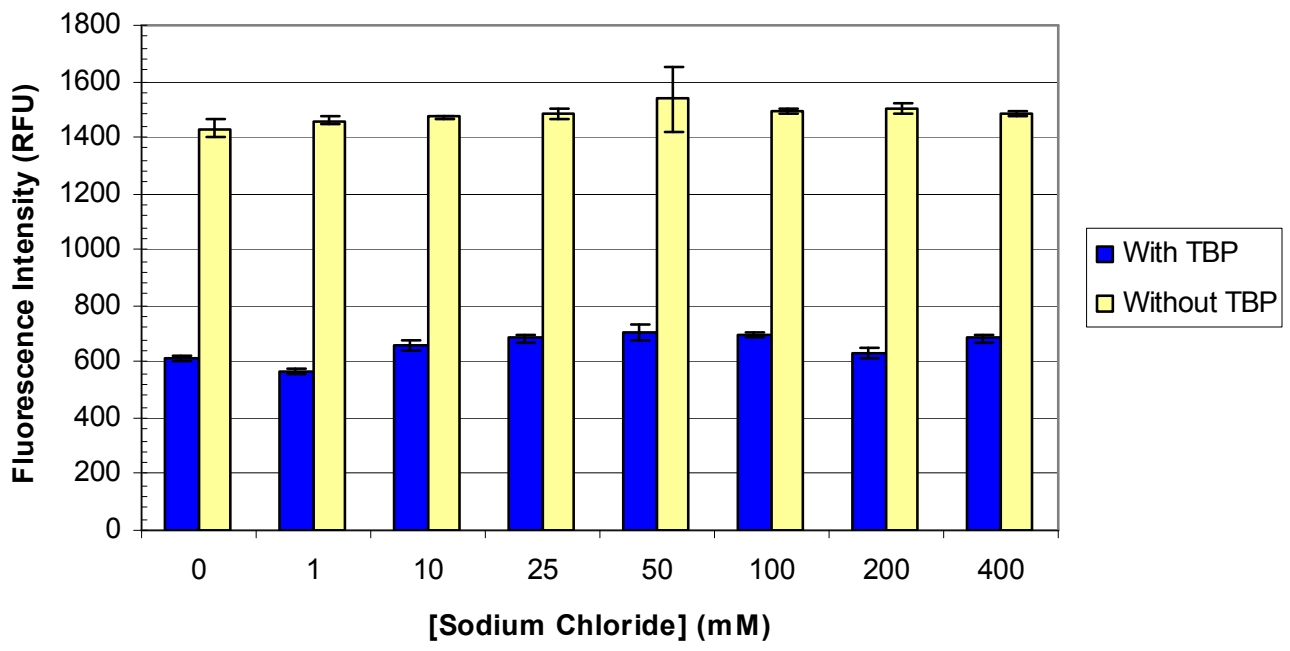

Figure S2. Effect of sodium chloride concentration on TBP binding to thiamine using equilibrium filtration assays. Each bar represents the average of triplicate determinations with error bars representing the standard deviation of these measurements. 
With an equivalent concentration of TBP and varying concentrations of thiamine, TMP, and TDP $(1,3$, and $6 \mu \mathrm{M})$, thiamine phosphate derivatives were bound more than thiamine. At a $1 \mu \mathrm{M}$ concentration, $68 \%$ thiamine was bound, $84 \%$ TMP, and $87 \%$ TDP was bound (Fig. S3).

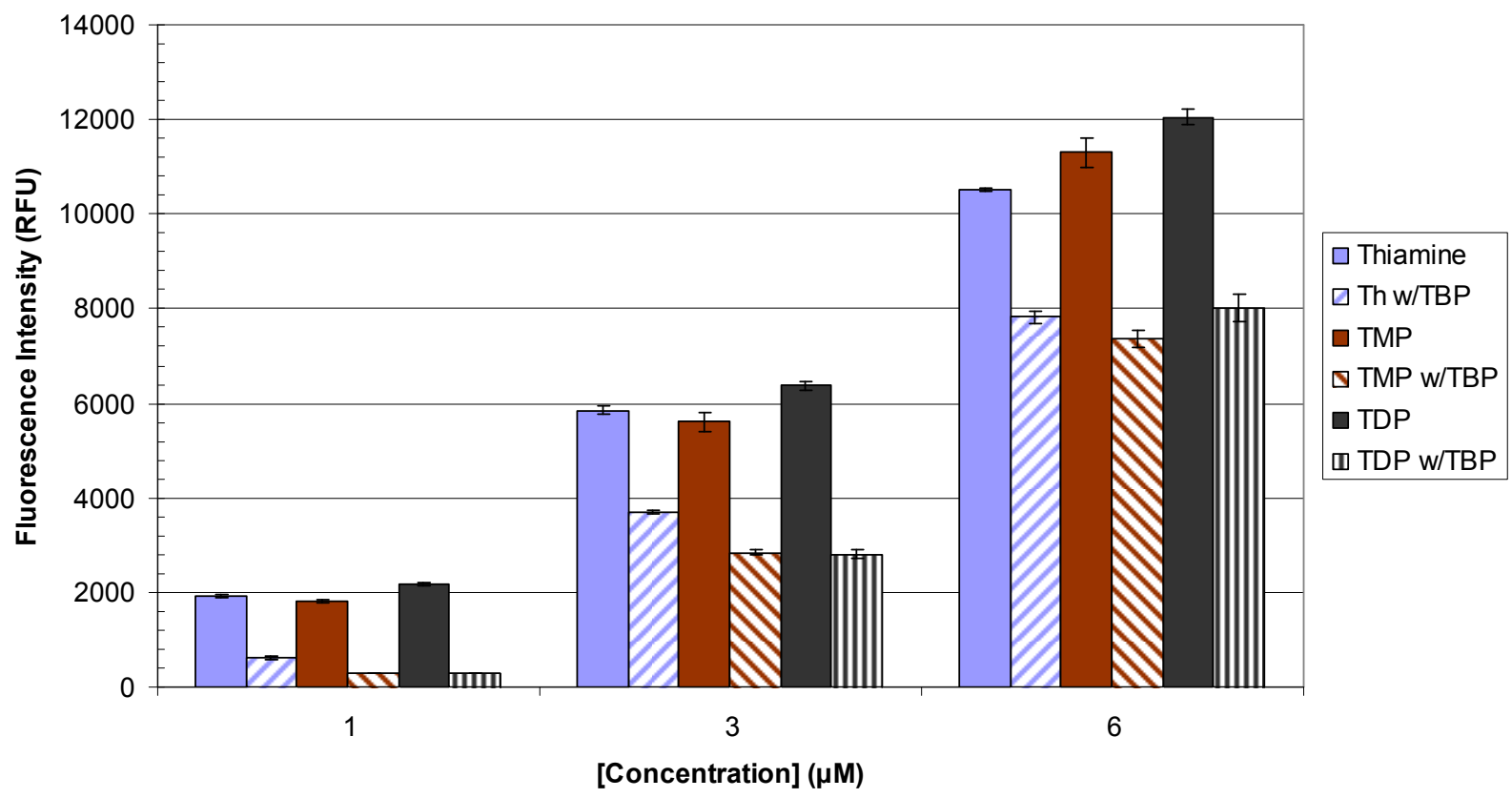

Figure S3. Direct binding of thiamine, TMP, and TDP by TBP using equilibrium filtration assays. Each bar represents the average of triplicate determinations with error bars representing the standard deviation of these measurements.

For competitive binding experiments, a 10-fold molar excess of thiamine derivatives or fragments (Table S1) were incubated with TBP and thiamine, followed by the same equilibrium filtration procedure described above. 
Table S1. Structures tested with direct or competitive binding.

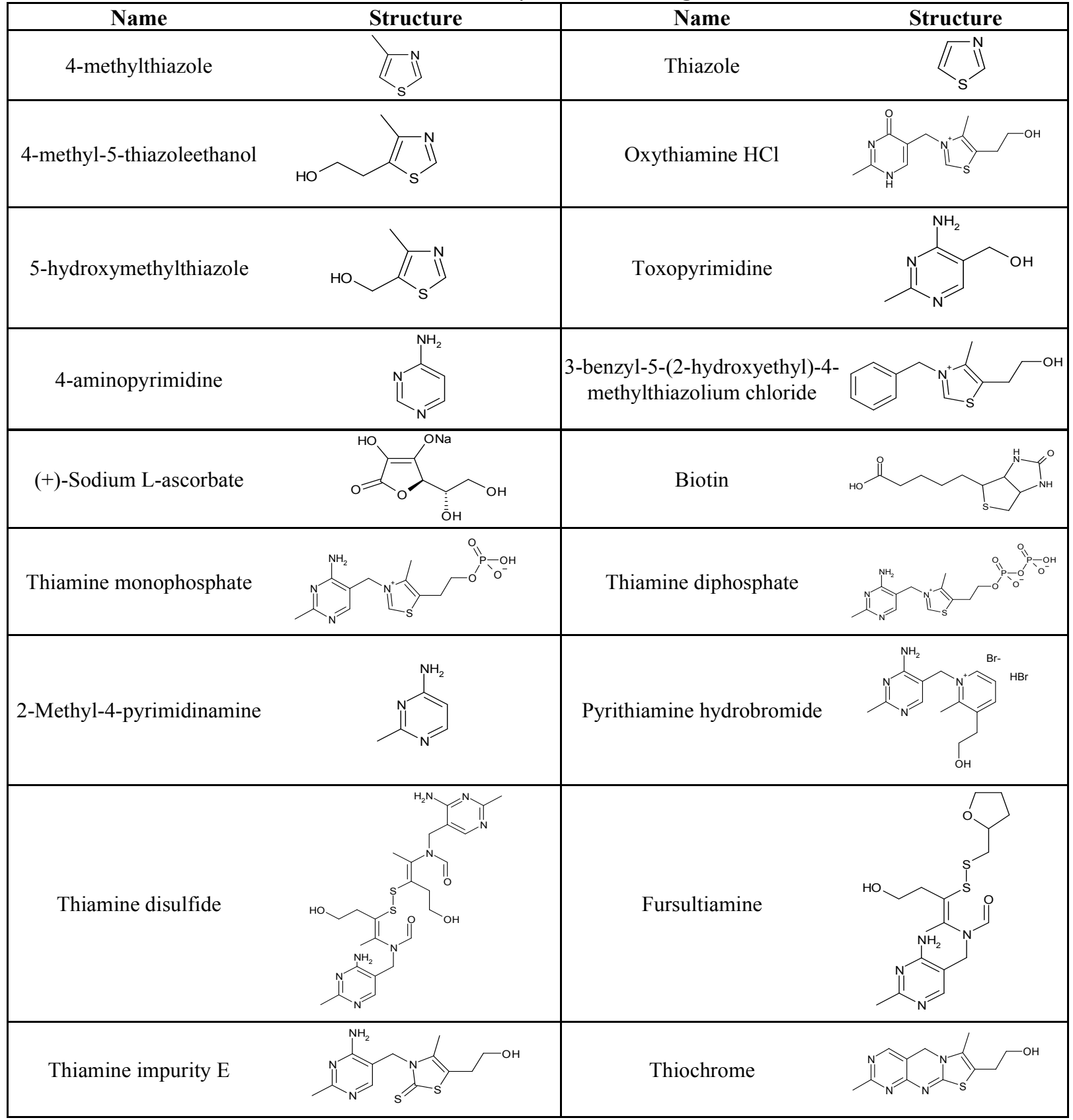

In competitive binding experiments, thiamine disulfide also appeared to yield some reduction in thiamine binding by TBP. However, as this lipophilic compound required dissolution in DMSO 
which is known to negatively impact binding, it was not possible to conclusively determine whether this was due to competitive binding or solvent effects.

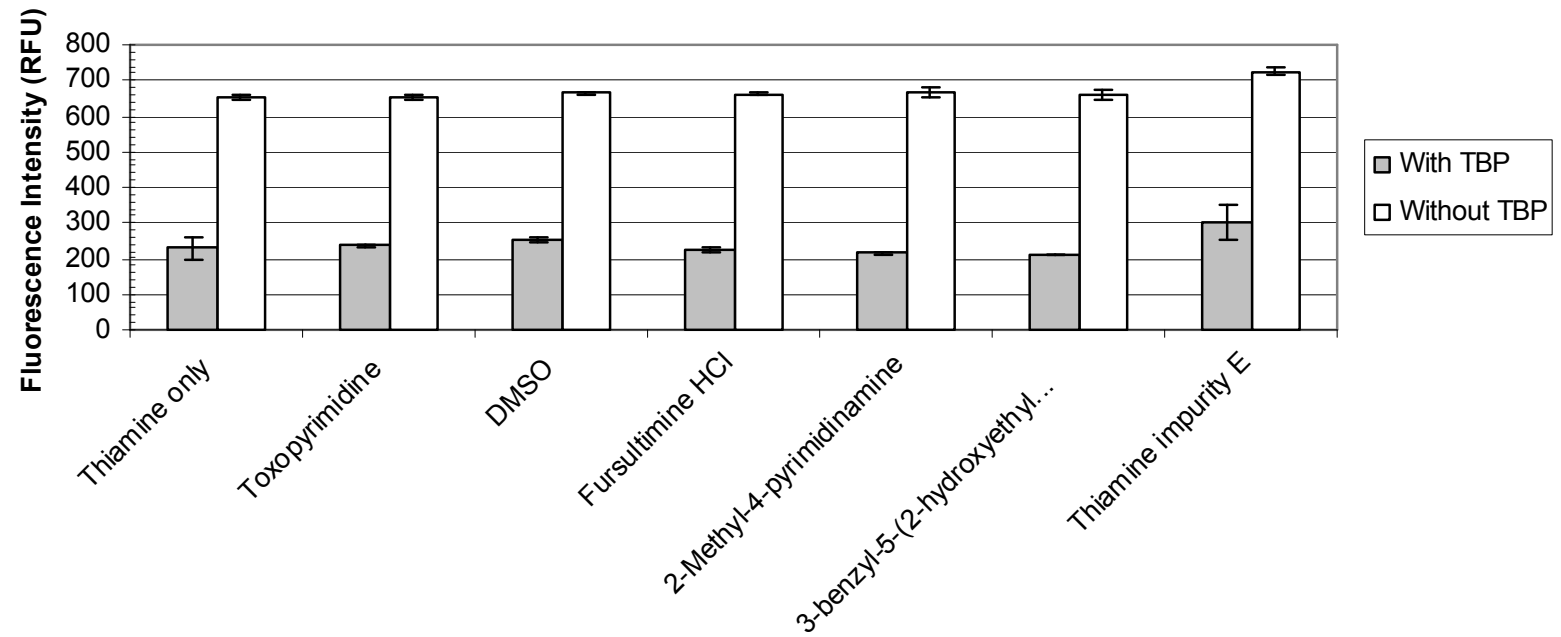

Figure S4. Specificity of the TBP to thiamine versus toxopyrimidine, DMSO, fursultiamine, 2methyl4-pyrimidinamine, 3-benzyl-5-(2-hydroxyethyl-4-methylthiazolium chloride), thiamine impurity $\mathrm{E}$ using equilibrium filtration assays. Each bar represents the average of triplicate determinations with error bars representing the standard deviation of these measurements. 


\section{Characterization of thiamine monosuccinate}

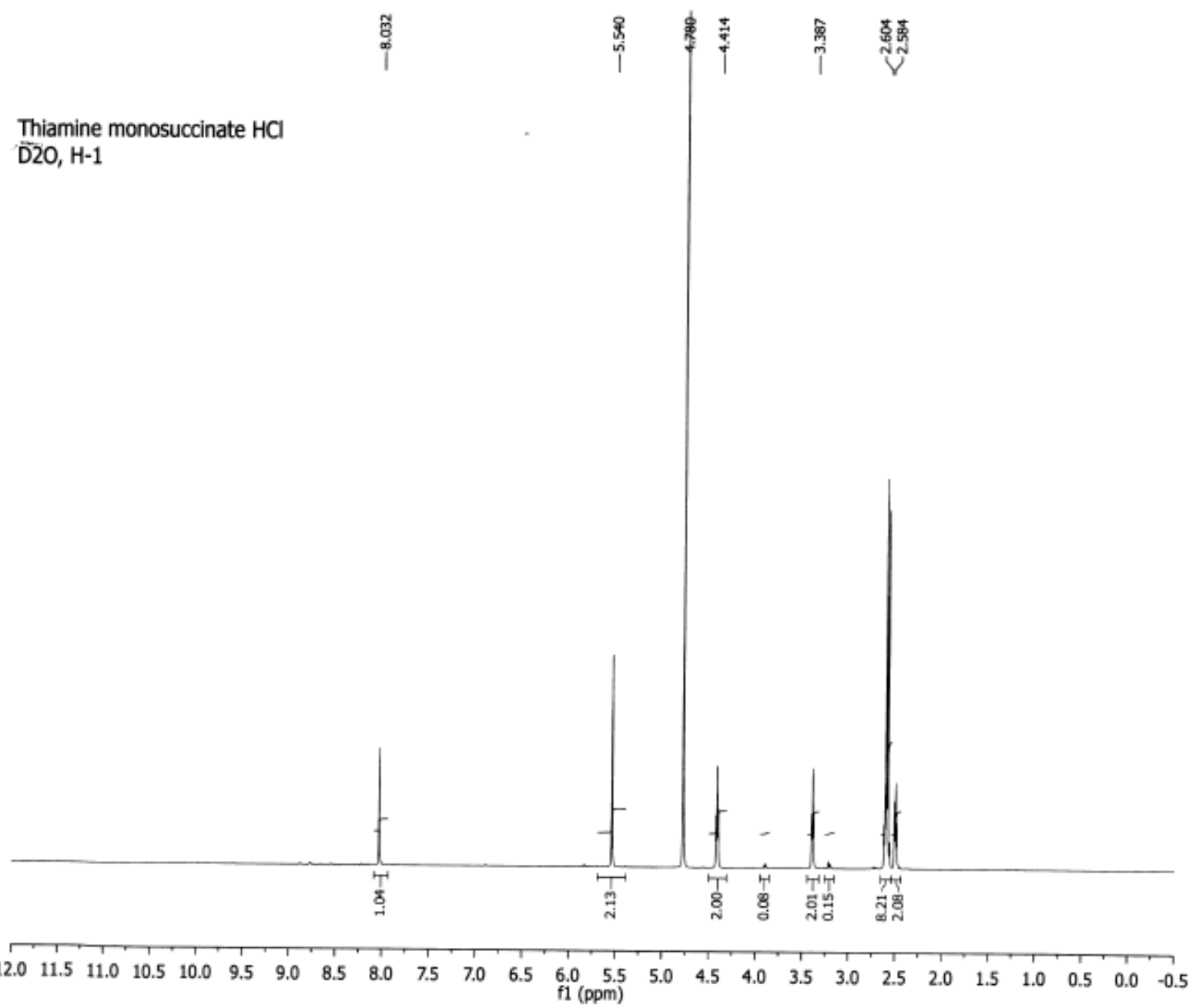

a.) 


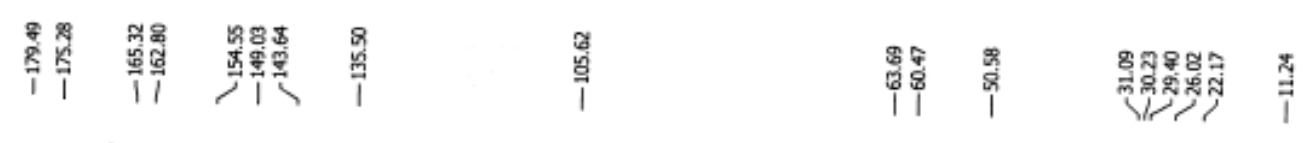

Thiamine monosuccinate $\mathrm{HCl}$

D2O, C-13
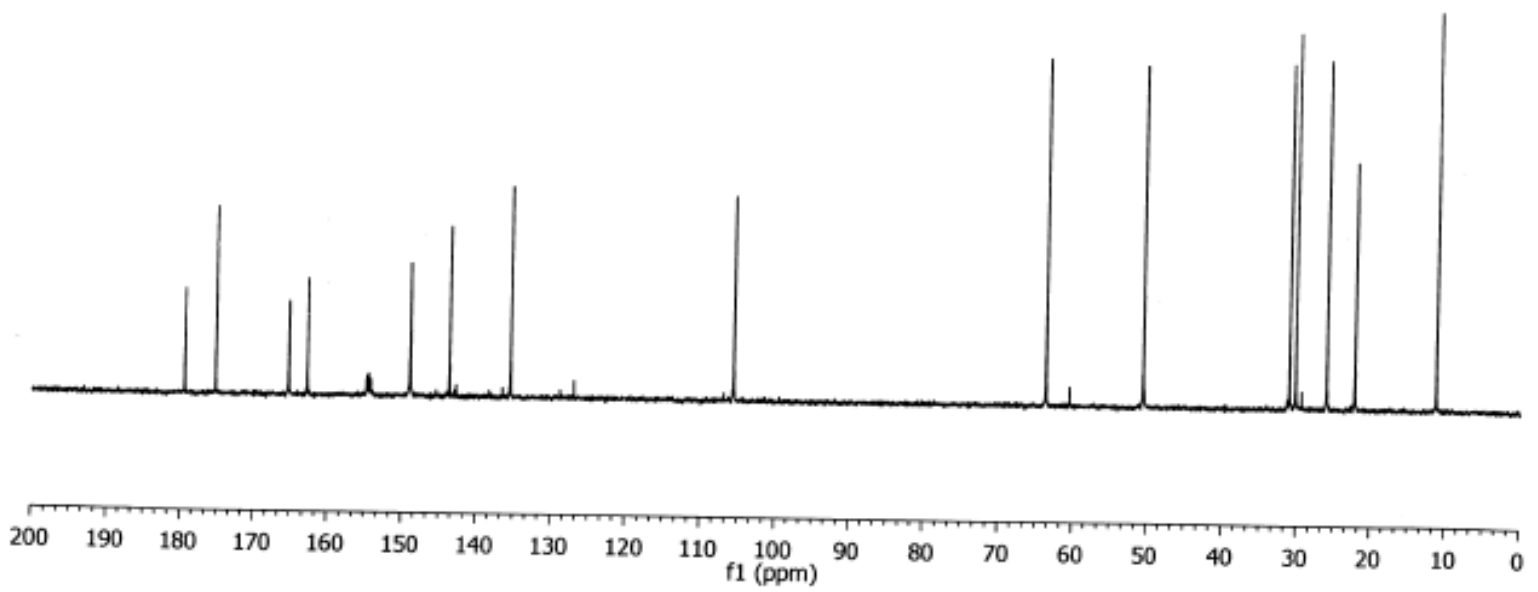

b.) 
Polarity/Scan Type: Positive Q1 Scan

Sample Name: Thiamine_monosuccinateHcl

Sample ID: TuneSample $\overline{I D}$

+Q1: 12 MCA scans from Sample 5 (Thiamine_monosuccinateHCl ...

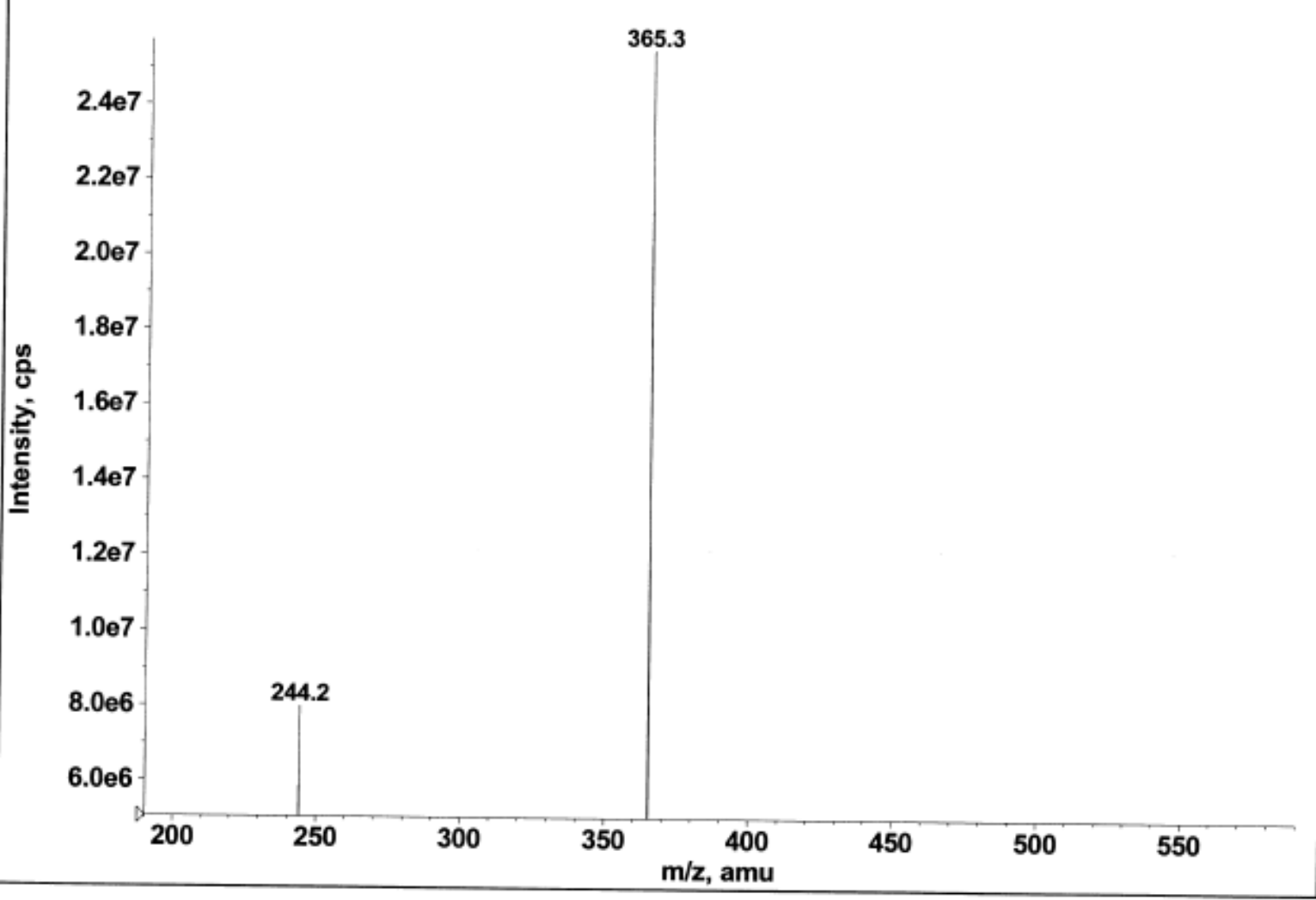

c.)

Figure S5. Characterization of thiamine monosuccinate by a.) ${ }^{1} \mathrm{H} N \mathrm{NR}$, b.) ${ }^{13} \mathrm{C} \mathrm{NMR}$, and c.) positive electron spray ionization MS 


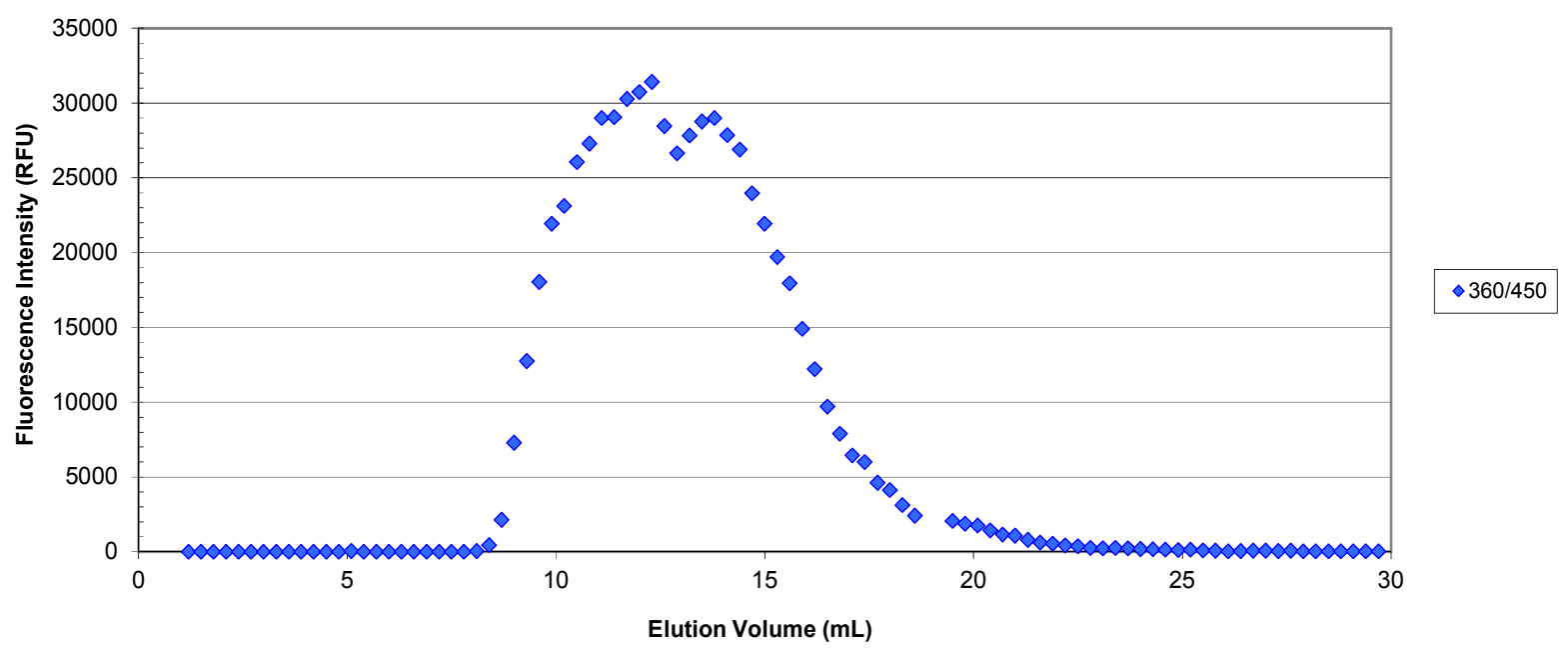

Figure S6. Size-exclusion chromatography of thiamine monosuccinate-PEG11-biotin and free thiamine from a Sephadex G10 column using 20 mM MES, $20 \mathrm{mM} \mathrm{NaCl}$, pH 6.5 as an eluent. Fractions were assayed by conversion to thiochrome to determine where the thiaminecontaining volumes eluted. Baseline resolution of the PEG11 conjugate $(1103 \mathrm{~g} / \mathrm{mol})$ from free thiamine monosuccinate $(365 \mathrm{~g} / \mathrm{mol}$ ) could not be obtained using this column, but pooled fractions with high concentrations of thiamine-PEG11-biotin could subsequently be dialyzed using 500 Da membranes to ensure that the purified conjugate was obtained. The material could be used without complete removal of residual thiaminine monosuccinate in the full competitive assay. The immobilization step using the biotin linkage via streptavidin allows for oriented immobilization and subsequent wash steps can remove any residual free thiamine monosuccinate prior to thiamine analysis.

\section{Competitive assay optimization}

To determine the optimal assay conditions as described in the materials and methods, various parameters including assay time (Fig. S7), liposome concentration, EDC concentration during TBP coupling (Fig. S8), ThSu-PEG11-biotin concentration (Fig. 2), pH (Fig. 4), blocking reagent, and blocking time were assessed.

By decreasing the incubation time from 90 minutes to 15 minutes, the calculated limit of detection decreased from $2 \mathrm{nM}$ to $0.2 \mathrm{nM}$ (Fig. S7). 


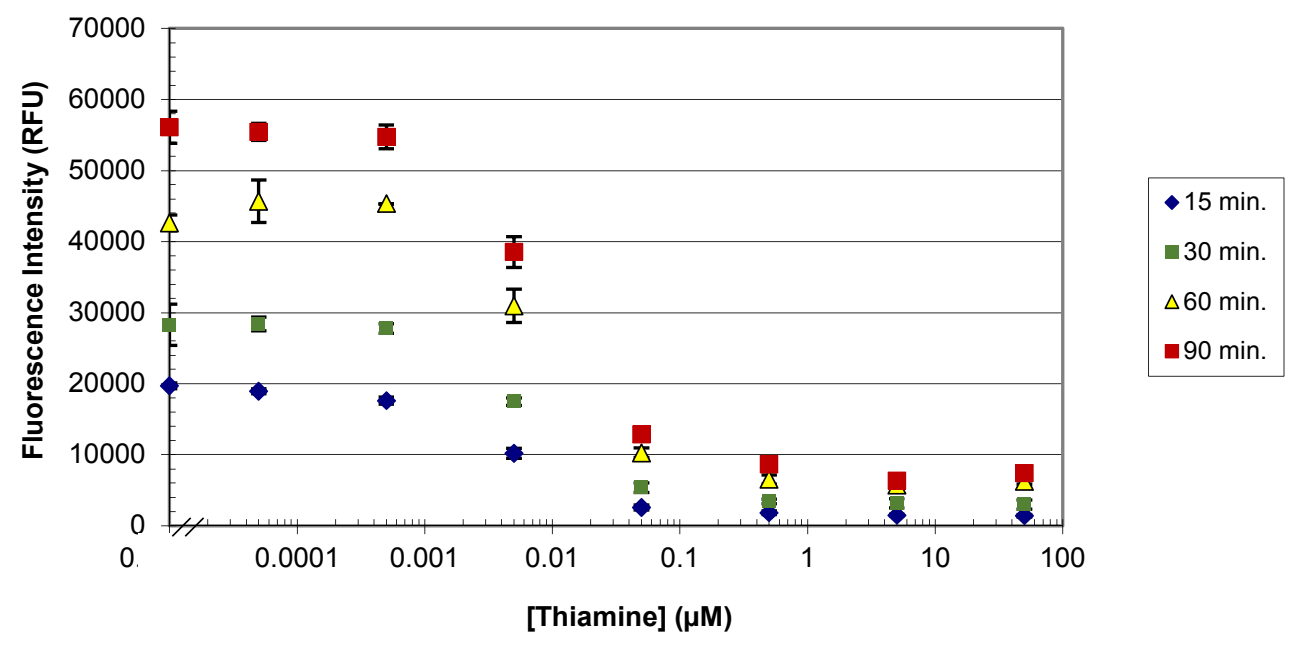

Figure S7. Effect of assay incubation time. Each point represents the average of triplicate determinations with error bars representing the standard deviation of these measurements.

With shorter incubation times, diffusion limitations permit fewer interactions of TBP liposomes with the thiamine monosuccinate-PEG11 surface which ultimately results in lower concentrations of thiamine needed for competition. The assay was more responsive to lower concentrations of thiamine at lower concentrations of liposomes. The liposome concentration allowing for a strong signal here $(6.25 \mu \mathrm{M})$ was lower than found optimal in previous assays (25-50 $\mu \mathrm{M})$, likely due to the high affinity of the TBP for the immobilized thiamine derivative. These $\mathrm{N}$-glutaryl (N-glut) liposomes interacted with the microtiter plate-immobilized ThSu-PEGbiotin only when conjugated to TBP (5-15 equivalents of EDC, Fig. S8.)

A minimum of $0.63 \mu \mathrm{M}$ of the thiamine-PEG11-conjugate was required for binding (Fig. 2). There was a marked on/off response observed between this concentration and the next lowest concentration tried $(0.31 \mu \mathrm{M})$. It is assumed that the signal resulting from interaction of TBP-conjugated liposomes with the surface is not a 1:1 relationship between a single liposome 
and a single thiamine-PEG11-conjugate, but rather from multivalent interactions between multiple TBP molecules on a single liposome and multiple immobilized thiamine-PEG11conjugates. Prior estimates of protein coverage using the maltose PBP coupled under identical conditions suggested $\sim 23 \mathrm{pmol} \mathrm{MBP} / \mathrm{mol}$ phospholipid, corresponding to $\sim 17$ molecules of PBP per $300 \mathrm{~nm}$ liposome ${ }^{6}$. The on/off response observed here may therefore be a function of having a requisite minimum number of appropriately spaced thiamine-PEG11-conjugate molecules to interact with multiple TBP molecules on a single liposome. In fact, an interesting avenue for future study will be to investigate how many attachment points are needed to permit interactions that withstand the washing conditions used in this assay.

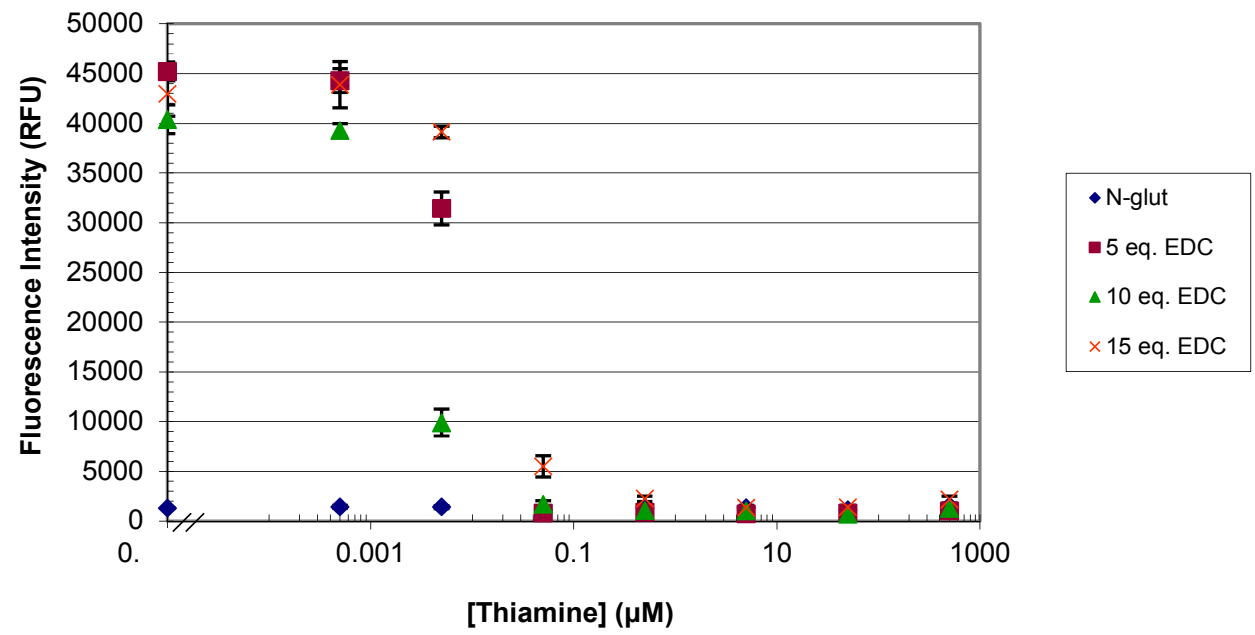

Figure S8. Effect of EDC concentration used during conjugation in terms of phospholipid concentration. Each point represents the average of triplicate determinations with error bars representing the standard deviation of these measurements. 


\section{Liposomal zeta potential}

One concern with this negatively-charged liposome formulation was the loss of electrostatic repulsion due to interaction with thiamine which is an organic cation. In early assay development where suboptimal surfaces were employed and high concentrations (mM) of solution-phase thiamine were used, significant non-specific binding of the liposomes was observed. This was attributed to a reduction of their surface charge due to interaction with thiamine. This observation was consistent with zeta potential measurements which indicated that this was a significant issue only at high thiamine concentrations (mM) (Fig. S9). Incubation of thiamine in the $\mu \mathrm{M}$ range with various formulations of liposomes did not yield detectable losses using equilibrium filtration assays. Such high concentrations are not present in environmental matrices, but it remains conceivable that some of the thiamine that is present at low levels may be lost due to electrostatic interaction with the lipids themselves, rather than via specific interaction with the attached TBP. 


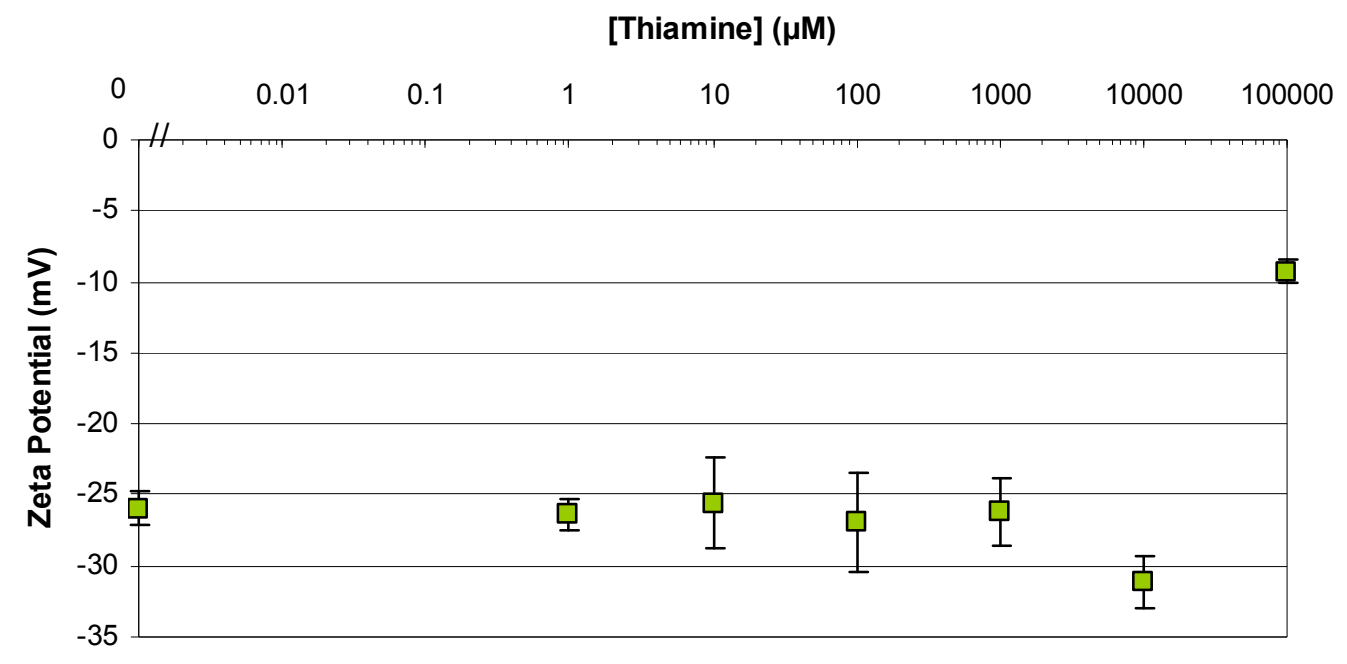

Figure S9. Effect of increasing thiamine concentrations on liposome zeta potential. Each point represents the average of triplicate determinations with error bars representing the standard deviation of these measurements.

\section{Comparison to commercially available thiamine ELISA}

The commercially available thiamine ELISA kit yielded a strong response to the supplied proprietary lyophilized standard (Fig. S10 top), but not thiamine supplied by commercial vendors (Fig. S10, bottom) even at markedly higher concentrations. 

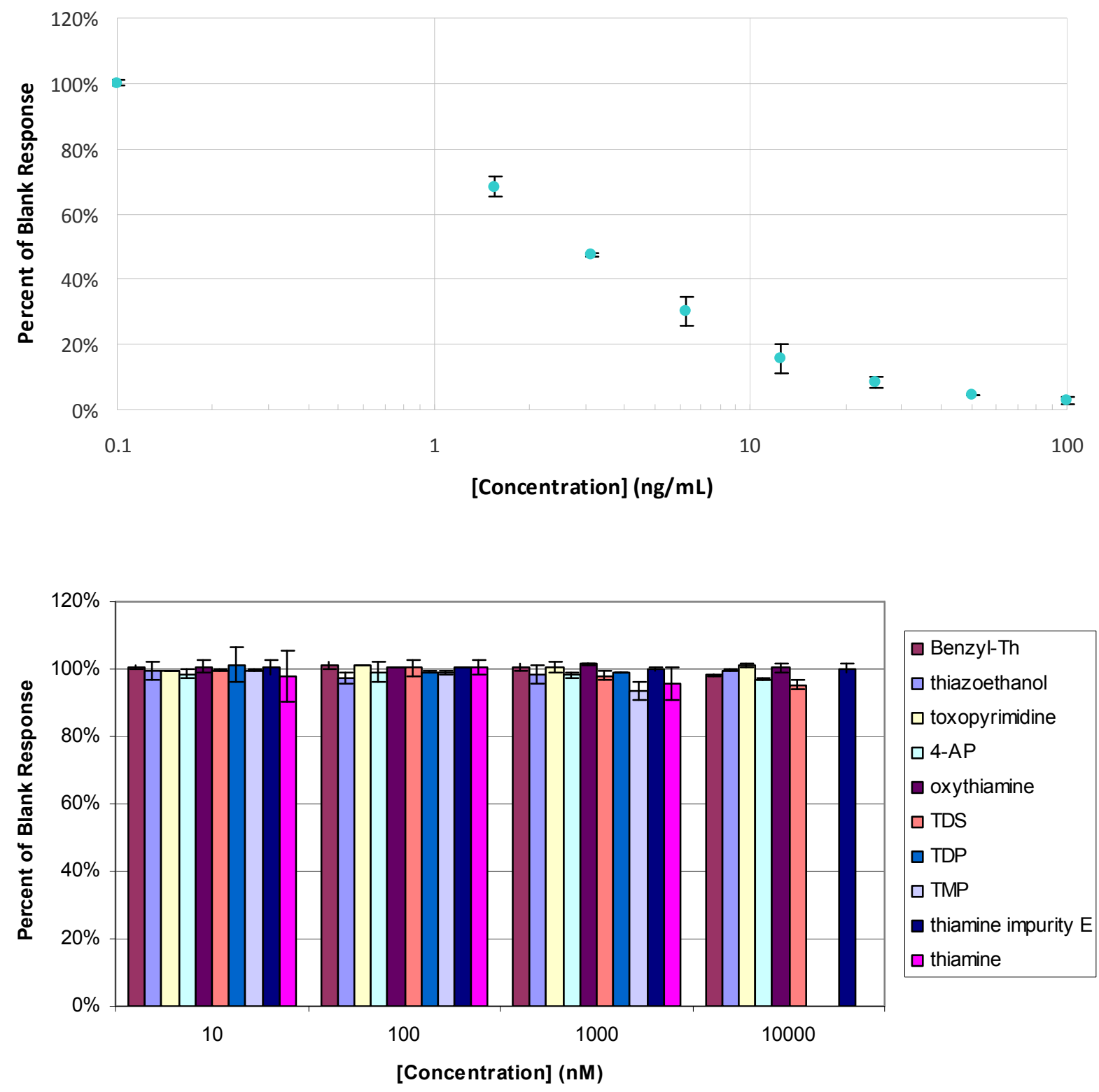

Figure S10. Thiamine ELISA kit response to proprietary lyophilized standard (top) and response to off-the-shelf thiamine versus TMP, TDP, thiamine disulfide, thiazoethanol, oxythiamine, toxopyrimidine, 4-aminopyrimidine, thiamine impurity E, and 3-benzyl-5-(2-hydroxyethyl)-4methylthiazolium chloride. Each bar represents the average of triplicate determinations with error bars representing the standard deviation of these measurements. 


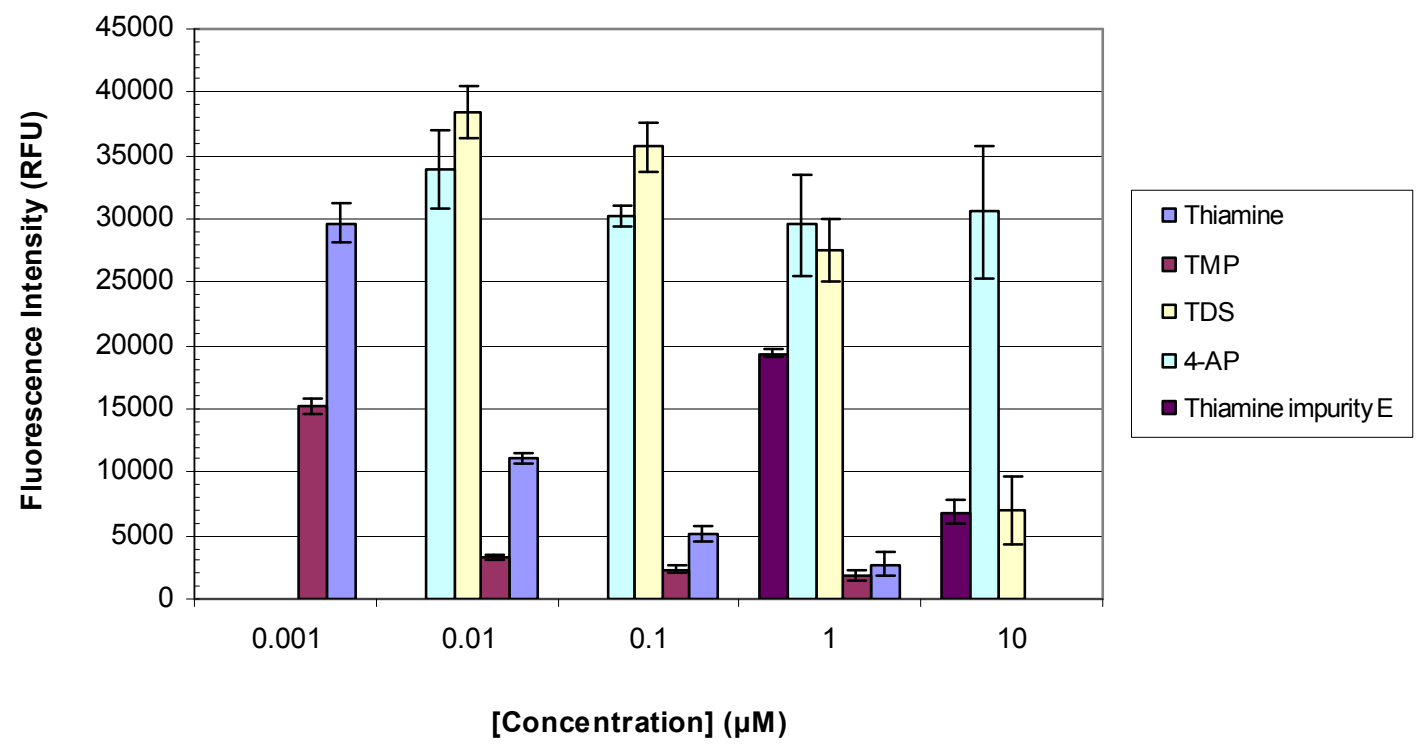

Figure S11. TBP-liposome assay response to thiamine versus TMP, TDP, thiamine disulfide, 4aminopyrimidine, and thiamine impurity E. Each bar represents the average of triplicate determinations with error bars representing the standard deviation of these measurements.

\section{References:}

1. Reeves SG, Rule GS, Roberts MA, Edwards AJ, Durst RA. Flow-injection liposome immunoanalysis (FILIA) for alachlor. Talanta. Oct 1994;41(10):1747-1753.

2. Szoka F, Papahadjopoulos, D. Procedure for preparation of liposomes with large internal aqueous space and high capture by reverse phase evaporation. Proc. Nat. Acad. Sci. USA. 1978;75:4194-4198.

3. Edwards KA, Curtis KL, Sailor JL, Baeumner AJ. Universal liposomes: preparation and usage for the detection of mRNA. Analytical and Bioanalytical Chemistry. Jul 2008;391(5):1689-1702.

4. Bartlett GR. Phosphorus Assay in Column Chromatography. Journal of Biological Chemistry. 1959;234(3):466-468.

5. Fiske $\mathrm{CH}$, Subbarow Y. The colorimetric determination of phosphorus. Journal of Biological Chemistry. Dec 1925;66(2):375-400.

6. Edwards KA, Baeumner AJ. Periplasmic binding protein-based detection of maltose using liposomes: a new class of biorecognition elements in competitive assays. Anal Chem. Mar 5 2013;85(5):2770-2778. 